\title{
Linezolid resistance in vancomycin resistant enterococci: a worrisome situation
}

Sir,

Vancomycin resistant enterococci (VRE) have emerged as important nosocomial pathogens since 1986. VRE have been associated with higher morbidity and mortality rates than vancomycin susceptible enterococci. ${ }^{1}$ Of over 50 species of Enterococcus, a genus of Gram-positive cocci arranged in pairs and short chains, E. faecalis is the most common cause of infections whereas $E$. faecium is the species exhibiting highest rate of antibiotic resistance. ${ }^{1}$ VRE have been implicated in varieties of infections such as bacteremia, infective endocarditis, intra-abdominal and pelvic infections, urinary tract infections, central nervous system infections and skin and skin structure infections. ${ }^{1}$ Since VRE exhibit multidrug resistance, there are very limited options for treatment of infections caused by them. One of the major treatment options is linezolid. The drug is the first member of oxazolidinones that received Food and Drug Administration (FDA) approval in 2000 as the last-resort drug for treatment of serious Gram-positive bacterial infections, including vancomycin-resistant enterococci (VRE), methicillin-resistant Staphylococcus aureus (MRSA) and multi-drug resistant Streptococcus pneumoniae infections. ${ }^{2}$ This bacteriostatic drug binds to rRNAs of both the $30 \mathrm{~S}$ and $50 \mathrm{~S}$ ribosomal subunits, inhibits formation of initiation complex and prevents the synthesis of bacterial protein. ${ }^{3}$ Though this drug has been very useful to treat serious infections caused by VRE, some strains of enterococci have already been found to exhibit resistance to this drug.

Resistance to linezolid was reported, for the first time, in 2001 , one year after its approval by FDA. ${ }^{4}$ Though rate of resistance is low, resistance among various isolates has now been documented from different parts of the world such as from Malaysia, Austria, China, Brazil and Africa. ${ }^{5-}$ 9 The most common mechanism of linezolid resistance described is the mutation in the $\mathrm{V}$ domain of the $23 \mathrm{~S}$ rRNA. ${ }^{6}$ Alteration of the target by the mutation disrupts the binding of oxazolidinones and other protein synthesis inhibitors. The other mechanisms of resistance that are reported include mutations in the sequence of genes encoding the riboproteins L3, L4 and L22 and plasmid mediated resistance due to cfr, optr A and poxtA gene. ${ }^{6}$ Linezolid resistance has been documented to disseminate through sex pheromone plasmid transfer in various clinical strains of Enterococcus faecalis. ${ }^{10}$ The possible risk factors associated with the resistance include transplants, complicated abdominal surgery, immunosuppression, use of vancomycin and broad spectrum antibiotics, chemotherapy induced neutropenia, previous or ongoing treatment with linezolid etc. ${ }^{5}$
The emergence of resistance to linezolid in VRE is a worrisome situation. There is no specific approved therapeutic drug currently available for treatment of infections caused by those organisms although few studies have highlighted the successful outcome using tigecycline and daptomycin individually. ${ }^{5-11}$ Therefore, control of indiscriminate use of this drug, continuous surveillance studies on its resistance and use of stringent infectioncontrol measures in the hospital to limit the spread of resistant strains are necessary.

\section{Rama Paudel ${ }^{1 *}$, Hari P. Nepal ${ }^{2}$}

${ }^{1}$ School of Biomedical Sciences, Trinity Medical Sciences University, Kingstown, St. Vincent and the Grenadines

${ }^{2}$ School of Medicine, Trinity Medical Sciences University, Kingstown, St. Vincent and the Grenadines

*Correspondence to Dr. Rama Paudel, E-mail: paudelrama2015@gmail.com

\section{REFERENCES}

1. O'Driscoll T, Crank CT. vancomycin-resistant enterococcal infections: epidemiology, clinical manifestations and optimal management. Infect Drug resist. 2015;8:217-30.

2. Leach KL, Brickner SJ, Noe MC, Miller PF. Linezolid, the first oxazolidinone antibacterial agent. Ann N Y Acad Sci. 2011;1222:49-54.

3. Batts DH. Linezolid-a new option for treating Grampositive infections. Oncology. 2000;14(8-6):23-9.

4. Bi R, Qin T, Fan W, Ma P, Gu B. The emerging problem of linezolid-resistant enterococci. J Global Antimirob Resist. 2018;11-9.

5. Chua WC, Zaidah AR. First reported cases of linezolid resistant vancomycin-resistant Enterococci in South-East Asia: A report of three cases and literature review. Proc Singapore Healthc. 2020;1-4.

6. Kerschner H, Cabal A, Hartl R, Machherndl-Spandl $S$, Allerberger F. Hospital outbreak caused by linezolid resistant Enterococcus faecium in Upper Austria. Antimicrob Resist Infect Control. 2019;8:150

7. Li P, Yang Y, Ding L, Xu X, Lin D. Molecular Investigations of Linezolid Resistance in Enterococci Optr A Variants from a Hospital in Shanghai. 2020;2020:(13):2711-6. 
8. De Almeida LM, De Araujo MRE, Iwasaki MR, Sacramento AG, Rocha D, Da Silva LP et al. Linezolid Resistance in Vancomycin-Resistant Enterococcus faecalis and Enterococcus faecium Isolates in a Brazilian Hospital. Antimicrob Agents Chemother. 2014;58(5):2993-4.

9. Raddaoui A, Chebbi Y, Tanfous FB, Mabrouk A, Achour W. First description of clinical linezolid resistant Enterococcus sp. in North Africa. J Global Antimirob Resist. 2020;21:169-70.

10. Zou J, Tank Z, Yan J, Liu H, Chen Y, Zhang D et al. Dissemination of linezolid resistance through sex pheromone plasmid transfer in Enterococcus faecalis. Front Microbiol. 2020;11:1185.

11. Mutschler M, Trojan S, Defosse JM, Helmers A, Probst C, Bouillon B et al. Severe sepsis caused by a linezolid-resistant Enterococcus faecium in a 10-yearold girl after multiple trauma. Int $\mathbf{J}$ Infect Dis. 2013;17(6):e466-7.

Cite this article as: Paudel R, Nepal HP. Linezolid resistance in vancomycin resistant enterococci: a worrisome situation. Int J Basic Clin Pharmacol 2021;10:464-5. 